\title{
A new relation between the condensation index of complex sequences and the null controllability of parabolic systems
}

\author{
Farid Ammar Khodja ${ }^{\mathrm{a}}$ Assia Benabdallah ${ }^{\mathrm{b}}$ Manuel González-Burgos ${ }^{\mathrm{c}}$ Luz de Teresa ${ }^{\mathrm{d}}$ \\ ${ }^{a}$ Laboratoire de Mathématiques, Université de Franche-Comté, 16 route de Gray, 25030 Besançon cedex, France \\ ${ }^{\mathrm{b}}$ Université de Provence, C.M.I. 39, rue F. Joliot Curie 13453 Marseille Cedex 13, France. \\ ${ }^{\mathrm{c}}$ Dpto. E.D.A.N., Universidad de Sevilla, Aptdo. 1160, 41080 Sevilla, Spain. \\ ${ }^{\mathrm{d}}$ Instituto de Matemáticas, Universidad Nacional Autónoma de México, Circuito Exterior, C.U. 04510 D.F., México.
}

Received *****; accepted after revision +++++

Presented by

\begin{abstract}
In this note we present a new result that relates the condensation index of a sequence of complex numbers with the null controllability of parabolic systems. We show that a minimal time is required for controllability. The results are used to prove the boundary controllability of some coupled parabolic equations. To cite this article: F. Ammar-Khodja, A. Benabdallah, M. González-Burgos, L. de Teresa, C. R. Acad. Sci. Paris, Ser. I 340 (2013).

\section{Résumé}

Une nouvelle rélation entre l'indice de condensation de séquences complexes et la nulle contrôlabilitée des systèmes paraboliques On annonce un résultat qui connecte l'indice de condensation des suites complexes et la nulle controlabilitée des systèmes paraboliques. On montre qu'un temps minimal est nécessaire pour controller, puis on voit le controle a zéro sur le bord de quelques équations paraboliques couplées. Pour citer cet article : F. Ammar-Khodja, A. Benabdallah, M. González-Burgos, L. de Teresa, C. R. Acad. Sci. Paris, Ser. I 340 (2013).
\end{abstract}

\section{Notation and main results}

Let $\mathbb{X}$ be a Hilbert space on $\mathbb{C}$ with norm and inner product respectively denoted by $\|\cdot\|$ and $(\cdot, \cdot)$. Let us consider $\left\{\phi_{k}\right\}_{k>1}$ a Riesz basis of $\mathbb{X}$ and denote $\left\{\psi_{k}\right\}_{k>1}$ the corresponding biorthogonal sequence to $\left\{\phi_{k}\right\}_{k \geq 1}$. Also consider a sequence $\Lambda=\left\{\lambda_{k}\right\}_{k \geq 1} \subset \mathbb{C}$, with $\lambda_{i} \neq \lambda_{k}$ for all $i \neq k$, satisfying for a $\delta>0$,

Email addresses: fammarkh@univ-fcomte.fr (Farid Ammar Khodja), assia@cmi.univ-mrs.fr (Assia Benabdallah), manoloburgos@us.es (Manuel González-Burgos), deteresa@matem.unam.mx (Luz de Teresa).

Preprint submitted to the Académie des sciences 


$$
\Re\left(\lambda_{k}\right) \geq \delta\left|\lambda_{k}\right|>0, \quad \forall k \geq 1, \quad \text { and } \quad \sum_{k \geq 1} \frac{1}{\left|\lambda_{k}\right|}<\infty
$$

Denote by $\mathbb{X}_{-1}$ the completion of $\mathbb{X}$ with respect to the norm: $\|y\|_{-1}:=\left(\sum_{k \geq 1} \frac{\left|\left(y, \psi_{k}\right)\right|^{2}}{\left|\lambda_{k}\right|^{2}}\right)^{1 / 2}$. Also the Hilbert space $\left(\mathbb{X}_{1},\|\cdot\|_{1}\right)$ is defined by $\mathbb{X}_{1}:=\left\{y \in \mathbb{X}:\|y\|_{1}<\infty\right\}$ with $\|y\|_{1}^{2}=\sum_{k \geq 1}\left|\lambda_{k}\right|^{2}\left|\left(y, \psi_{k}\right)\right|^{2}$. Furthermore, let $\mathcal{A}: \mathcal{D}(\mathcal{A})=\mathbb{X}_{1} \subset \mathbb{X} \rightarrow \mathbb{X}$ be the operator given by:

$$
\mathcal{A}=-\sum_{k \geq 1} \lambda_{k}\left(\cdot, \psi_{k}\right) \phi_{k}
$$

Let us fix $T>0$ a real number and $\mathcal{B} \in \mathcal{L}\left(\mathbb{C}, \mathbb{X}_{-1}\right)$ (so $\left.\mathcal{B}^{*} \in \mathcal{L}\left(\left(\mathbb{X}_{-1}\right)^{\prime}, \mathbb{C}\right) \equiv \mathbb{X}_{-1}\right)$. We consider:

$$
y^{\prime}=\mathcal{A} y+\mathcal{B} u \quad \text { on }(0, T) ; \quad y(0)=y_{0} \in \mathbb{X} .
$$

In System $(3), u \in L^{2}(0, T ; \mathbb{C})$ is the control which acts on the system by means of the operator $\mathcal{B}$. We assume that $\mathcal{B}$ is an admissible control operator for the semigroup generated by $\mathcal{A}$, i.e., for a positive time $T^{*}$ one has $\mathrm{R}\left(L_{T^{*}}\right) \subset \mathbb{X}$, where $L_{T} u=\int_{0}^{T} e^{(T-s) \mathcal{A}} \mathcal{B} u(s) d s$. System (3) is approximately controllable in $\mathbb{X}$ at time $T>0$ if for every $y_{0} \in \mathbb{X}, \mathcal{R}(T)=\left\{y(T)=e^{T \mathcal{A}} y_{0}+L_{T} u\right.$ with $\left.u \in L^{2}(0, T ; \mathbb{C})\right\}$ is dense in $\mathbb{X}$ and System (3) is null controllable in $\mathbb{X}$ at time $T>0$ if for all $y_{0} \in \mathbb{X}, 0 \in \mathcal{R}(T)$. It is well-known that the controllability properties of System (3) amount to appropriate properties of the so-called adjoint system to System (3). This adjoint system has the form:

$$
-\varphi^{\prime}=\mathcal{A}^{*} \varphi \quad \text { on }(0, T) ; \quad \varphi(T)=\varphi_{0} \in \mathbb{X} .
$$

Observe that, for any $\varphi_{0} \in \mathbb{X}$, System (4) admits a unique weak solution $\varphi \in C^{0}([0, T] ; \mathbb{X})$. Classical results (see e.g. [6, Theorem 11.2.1]) imply:

Theorem 1.1 Assume that $\mathcal{B} \in \mathcal{L}\left(\mathbb{C}, \mathbb{X}_{-1}\right)$ is an admissible control operator for the semigroup $\left\{e^{t \mathcal{A}}\right\}_{t>0}$ generated by $\mathcal{A}$, with $\mathcal{A}$ given by (2), and $\Lambda=\left\{\lambda_{k}\right\}_{k>1}$ is a complex sequence satisfying (1). Then, system (3) is approximately controllable in $\mathbb{X}$ at time $T$ if and only if

$$
b_{k}:=\mathcal{B}^{*} \psi_{k} \neq 0, \quad \forall k \geq 1
$$

Moreover, (3) is null controllable in $\mathbb{X}$ at time $T$ if and only if there exists a constant $C_{T}>0$ such that

$$
\sum_{k \geq 1} e^{-2 T \Re\left(\lambda_{k}\right)}\left|a_{k}\right|^{2} \leq C_{T} \int_{0}^{T}\left|\sum_{k \geq 1} \bar{b}_{k} e^{-\lambda_{k}(T-t)} a_{k}\right|^{2}, \quad \forall\left\{a_{k}\right\}_{k \geq 1} \in \ell^{2}(\mathbb{C}) .
$$

Our main result reads as follows:

Theorem 1.2 Assume that $\mathcal{B} \in \mathcal{L}\left(\mathbb{C}, \mathbb{X}_{-1}\right)$ is an admissible control operator for the semigroup $\left\{e^{t \mathcal{A}}\right\}_{t>0}$ and $\Lambda=\left\{\lambda_{k}\right\}_{k \geq 1}$ is a complex sequence satisfying respectively (5) and (1). For $z \in \mathbb{C}$, let us introduce $E(z)=\prod_{k=1}^{\infty}\left(1-\frac{z^{2}}{\lambda_{k}^{2}}\right)$ and $T_{0}=\limsup \left(\frac{\log \frac{1}{\left|b_{k}\right|}}{\Re\left(\lambda_{k}\right)}+\frac{\log \frac{1}{\left|E^{\prime}\left(\lambda_{k}\right)\right|}}{\Re\left(\lambda_{k}\right)}\right)$. Then System (3) is null controllable for $T>T_{0}$ and is not null controllable for $T<T_{0}$.

The index of condensation of a sequence $\Lambda=\left\{\lambda_{k}\right\}_{k \geq 1} \subset \mathbb{C}$ satisfying (1) is the real number $c(\Lambda)=$ $\limsup \frac{\log \frac{1}{E^{\prime}\left(\lambda_{k}\right) \mid}}{\Re\left(\lambda_{k}\right)}$, where the function $E$ is given in Theorem 1.2. The condensation index is related to the overconvergence of Dirichlet series (see [5]). Observe that when $\lim \frac{\log \left|b_{k}\right|}{\Re\left(\lambda_{k}\right)}=0$, then, $T_{0}=c(\Lambda)$. 


\section{Idea of the proof of Theorem 1.2}

The proof is technical and long and the details are given in [2]. For the proof of the positive result we transform the control problem into a problem of moments. So we need to study the existence of biorthogonal families to complex exponentials and study some properties of these families. We have the following result:

Theorem 2.1 Let $\Lambda=\left\{\lambda_{k}\right\}_{k \geq 1} \subset \mathbb{C}$ be a sequence satisfying (1) and fix $T \in(0, \infty]$. Let $A(\Lambda, T)=$ ${\overline{\operatorname{span}}\left\{e^{-\lambda_{k} t}: k \geq 1\right\}}^{L^{2}(0, T ; \mathbb{C})}$. Then, there exists a biorthogonal family $\left\{q_{k}\right\}_{k \geq 1} \subset A(\Lambda, T)$ to $\left\{e^{-\lambda_{k} t}\right\}_{k \geq 1}$ such that for any $\varepsilon>0$ one has

$$
C_{1, \varepsilon} \frac{e^{-\varepsilon \Re\left(\lambda_{k}\right)}}{\left|E^{\prime}\left(\lambda_{k}\right)\right|} \leq\left\|q_{k}\right\|_{L^{2}(0, T ; \mathbb{C})} \leq C_{2, \varepsilon} \frac{e^{\varepsilon \Re\left(\lambda_{k}\right)}}{\left|E^{\prime}\left(\lambda_{k}\right)\right|}, \quad \forall k \geq 1,
$$

where $E$ is the function given in Theorem 1.2 and $C_{1, \varepsilon}, C_{2, \varepsilon}>0$ are constants only depending on $\varepsilon, \Lambda$ and $T$.

The null controllability problem for System (3) reduces to the following moment problem: Find $u \in$ $L^{2}(0, T ; \mathbb{C})$ such that, for $b_{k}$ given by $(5)$, we have $\bar{b}_{k} \int_{0}^{T} e^{-\lambda_{k} t} u(T-t) d t=-e^{-\lambda_{k} T}\left(y_{0}, \psi_{k}\right), \quad \forall k \geq 1$. We can solve this equality using the characterization of the biorthogonal family given above. So: $u(t)=$ $v(T-t)=-\sum_{k \geq 1} \frac{e^{-\lambda_{k} T}}{\bar{b}_{k}}\left(y_{0}, \psi_{k}\right) \bar{q}_{k}(T-t)$. It follows that if $T>T_{0}$, with $T_{0}$ given in Theorem 1.2 , the previous series is absolutely convergent in $L^{2}(0, T ; \mathbb{C})$ and thus $u \in L^{2}(0, T ; \mathbb{C})$. Indeed, if we choose $\varepsilon \in$ $\left(0, T-T_{0}\right)$, then $(7)$ leads to: $\left\|\frac{e^{-\lambda_{k} T}}{\bar{b}_{k}}\left(y_{0}, \psi_{k}\right) \bar{q}_{k}\right\|_{L^{2}(0, T ; \mathbb{C})}^{2} \leq C_{\varepsilon} e^{-2 \Re\left(\lambda_{k}\right)\left(T-T_{0}-\varepsilon\right)}\left|\left(y_{0}, \psi_{k}\right)\right|^{2}, \forall k \geq k_{\varepsilon} \geq 1$.

We prove that System (3) is not null controllable at time $T$, when $T<T_{0}$, showing that inequality (6) does not hold. Without loss of generality, we can assume that the sequence $\Lambda=\left\{\lambda_{k}\right\}_{k>1} \subset \mathbb{C}$ is normally ordered, i.e., $\left|\lambda_{k}\right| \leq\left|\lambda_{k+1}\right|$ for any $k \geq 1$ and $\arg \left(\lambda_{k}\right)<\arg \left(\lambda_{k+1}\right)$ when $\left|\lambda_{k}\right|=\left|\lambda_{k+1}\right|$. The negative part of Theorem 1.2 is a consequence of the following result:

Theorem 2.2 Let $\Lambda=\left\{\lambda_{k}\right\}_{k>1} \subset \mathbb{C}$ be a normally ordered sequence satisfying condition (1). Then, there exists a sequence of sets $\Delta=\left\{G_{k}\right\}_{k \geq 1}$ such that $\cup_{k \geq 1} G_{k} \cap \Lambda=\Lambda$ and for any subsequence $\left\{\lambda_{n_{k}}\right\}_{k \geq 1} \subseteq \Lambda$, one has:

$$
\lim \left(\frac{\log \frac{1}{\left|E^{\prime}\left(\lambda_{n_{k}}\right)\right|}}{\Re\left(\lambda_{n_{k}}\right)}-\frac{1}{\Re\left(\lambda_{n_{k}}\right)} \log \left|\frac{q_{k} !}{P_{D_{k}}^{\prime}\left(\lambda_{n_{k}}\right)}\right|\right)=0
$$

where $\left\{D_{k}\right\}_{k \geq 1} \subseteq \Delta$ is a subsequence of sets satisfying $\lambda_{n_{k}} \in D_{k}$ and $q_{k}+1$ is the cardinal of the set $D_{k} \cap \Lambda$. In the previous equality $P_{A}$ is the polynomial function $P_{A}(z)=\prod_{\lambda \in A}(z-\lambda)$.

Suppose that the observability inequality (6) holds. Using the previous result, we introduce $a_{n}^{(k)}=$ $\frac{p_{k} !}{\bar{b}_{n} P_{G_{k}}^{\prime}\left(\lambda_{n}\right)}$ if $\lambda_{n} \in G_{k}$ and 0 otherwise $\left(p_{k}+1\right.$ is the cardinal of $\left.G_{k} \cap \Lambda\right)$. Clearly, the (finite) sequence $\left\{a_{n}^{(k)}\right\}_{n \geq 1}$ lies in $\ell^{2}(\mathbb{C})$. From (6), we can write:

$$
\sigma_{k}^{(1)}:=\sum_{\lambda_{n} \in G_{k}}\left|\frac{p_{k} !}{\bar{b}_{n} P_{G_{k}}^{\prime}\left(\lambda_{n}\right)} e^{-\lambda_{n} T}\right|^{2} \leq C_{T} \int_{0}^{T}\left|\sum_{\lambda_{n} \in G_{k}} \frac{p_{k} !}{P_{G_{k}}^{\prime}\left(\lambda_{n}\right)} e^{-\lambda_{n} t}\right|^{2} d t:=\sigma_{k}^{(2)}, \quad \forall k \geq 1 .
$$

Using the Lebesgue Theorem, it can be shown that $\lim \sigma_{k}^{(2)}=0$. On the other hand, from the definition of $T_{0}$ (see Theorem 1.2) and (8), there exists $\left\{n_{k}\right\}_{k \geq 1}$ such that $T_{0}=\lim \frac{1}{\Re\left(\lambda_{n_{k}}\right)}\left(\log \left|\frac{1}{b_{n_{k}}}\right|+\log \left|\frac{q_{k} !}{P_{D_{k}}^{\prime}\left(\lambda_{n_{k}}\right)}\right|\right)$, where $\left\{D_{k}\right\}_{k \geq 1} \subseteq \Delta$ is a subsequence of sets satisfying $\lambda_{n_{k}} \in D_{k}$, for any $k$, and $q_{k}+1$ is the cardinal of 
the set $D_{k} \cap \Lambda$. Observe that $\sigma_{n_{k}}^{(1)} \geq\left|\frac{q_{k} !}{\bar{b}_{n_{k}} P_{D_{k}}^{\prime}\left(\lambda_{n_{k}}\right)} e^{-\lambda_{n_{k}} T}\right|^{2}=e^{2 \Re\left(\lambda_{n_{k}}\right)\left[\frac{1}{\Re\left(\lambda_{n_{k}}\right)}\left(\log \left|\frac{1}{b_{n_{k}}}\right|+\log \left|\frac{q_{k} !}{P_{D_{k}}^{\prime}\left(\lambda_{n_{k}}\right)}\right|\right)-T\right]}$.

This last inequality shows $\lim \sigma_{n_{k}}^{(1)}=\infty$. This contradicts (9). For the details, see [2].

\section{An application: A boundary controllability problem}

For $T>0$ and $Q=(0, \pi) \times(0, T)$, consider the one-dimensional controlled (non-scalar) system

$$
\left\{\frac{\partial y}{\partial t}-\left(\left(\begin{array}{ll}
1 & 0 \\
0 & d
\end{array}\right) \frac{\partial^{2}}{\partial x^{2}}+\left(\begin{array}{ll}
0 & 1 \\
0 & 0
\end{array}\right)\right) y=0, \text { in } Q \quad y(0, \cdot)=\left(\begin{array}{l}
b_{1} \\
b_{2}
\end{array}\right) v, \quad y(\pi, \cdot)=0 \quad \text { on }(0, T),\right.
$$

and initial datum $y(\cdot, 0)=y_{0}$ in $(0, \pi), y_{0} \in H^{-1}\left(0, \pi ; \mathbb{R}^{2}\right)$ and $d>0$. Observe that $v \in L^{2}(0, T)$ is a scalar boundary control which acts on the Dirichlet boundary condition of the state at point $x=0$ by means of the vector $\left(b_{1}, b_{2}\right)^{\top}$. The aim is to control the whole system (two states) with a control force $v$.

The control problem (10) has been completely solved in [3] when $d=1$. For a general system of $n \geq 2$ coupled equations with $M=I_{n}$, see [1]. The controllability problem for System (10) when $d \neq 1$ is more intricate and only few results are known. For $b_{1}=0$ and $b_{2}=1$ : Firstly, System (10) is approximately controllable in $H^{-1}\left(0, \pi ; \mathbb{R}^{2}\right)$ at time $T$ if and only if $\sqrt{d} \notin \mathbb{Q}$ (see [3]). Secondly, there exists $d \in(0, \infty)$ with $\sqrt{d} \notin \mathbb{Q}$ such that System (10) is not null controllable at any time $T>0$ (see [4]).

To our knowledge and apart from the previous results, the controllability properties of System (10) are completely open in the case $d \neq 1$. As a consequence of Theorem 1.2, we have:

Theorem 3.1 Assume $d \neq 1$ and let $c\left(\Lambda_{d}\right)$ be the index of condensation of the sequence $\Lambda_{d}:=\left\{k^{2}, d k^{2}\right\}_{k \geq 1}$. Then, (i) System (10) is approximately controllable in $\mathbb{X}=H^{-1}\left(0, \pi ; \mathbb{R}^{2}\right)$ at any time $T>0$ if and only if $\sqrt{d} \notin \mathbb{Q}$ and $b_{2}\left[(d-1) k^{2} b_{1}+d b_{2}\right] \neq 0$. (ii) System (10) is null controllable in $\mathbb{X}$ at any time $T>c\left(\Lambda_{d}\right)$ and is not null controllable in $\mathbb{X}$ for $T<c\left(\Lambda_{d}\right)$. (iii) For any $\tau_{0} \in[0, \infty]$, there exists $d \in(0, \infty)$ with $\sqrt{d} \notin \mathbb{Q}$ such that $c\left(\Lambda_{d}\right)=\tau_{0}$.

\section{Acknowledgements}

The authors where want to thank the partial support of grants: ANR-07-JCJC-0139-01 (Agence Nationale de la Recherche (France)), MTM2010-15592 (D.G.E.S. (Spain)), IN108211 (PAPIIT(Mexico)).

\section{References}

[1] F. Ammar-Khodja, A. Benabdallah, M. González-Burgos, L. de Teresa, The Kalman condition for the boundary controllability of coupled parabolic systems. Bounds on biorthogonal families to complex matrix exponentials, J. Math. Pures Appl. (9) 96 (2011), no. 6, 555-590.

[2] F. Ammar-Khodja, A. Benabdallah, M. González-Burgos, L. de Teresa, Optimal time for the null controllability of parabolic systems: the effect of the condensation index of complex sequences, submitted.

[3] E. Fernández-Cara, M. González-Burgos, L. de Teresa, Boundary controllability of parabolic coupled equations, J. Funct. Anal. 259 (2010), no. 7, 1720-1758.

[4] F. Luca, L. de Teresa, System controllability and Diophantine approximations, SeMA Journal 61 (2013), no. 1, 1-17.

[5] J. R. Shackell, Overconvergence of Dirichlet series with complex exponents, J. Analyse Math. 22 (1969), 135-170.

[6] M. Tucsnak, G. Weiss, Observation and Control for Operator Semigroups, Birkhäuser Advanced Texts: Basler Lehrbücher, Birkhäuser Verlag, Basel, 2009. 